\title{
Receptividad de los ganaderos aledaños a los ecosistemas de humedal en el establecimiento y manejo de fincas pecuarias integradas
}

\section{Receptivity of Livestock Farmers Adjacent to Wetland Ecosystems in the Establishment and Management of Integrated Livestock Farms}

\begin{abstract}
María Marcela Vargas Sibaja Universidad Nacional Sede Regional Chorotega, Campus Nicoya, Centro Mesoamericano de Desarrollo del Trópico Seco Heredia, Costa Rica maria.vargas.sibaja@una.cr

William Gómez Solís Universidad Nacional

Sede Regional Chorotega, Campus Nicoya, Centro Mesoamericano de Desarrollo del Trópico Seco Heredia, Costa Rica william.gomez.solis@una.cr
\end{abstract} Recibido 02/06/2017 Aceptado 05/09/2017

Resumen. El objetivo de la investigación fue abordar la receptividad, aplicación y utilidad de los pequeños ganaderos en el proyecto Establecimiento y Manejo de Fincas Pecuarias Integradas, el cual inició en el 2014 y finalizó en diciembre de 2016, en las fincas aledañas al ecosistema de humedal de Mata Redonda, en Rosario de Nicoya. El proyecto nació gracias a la necesidad de extensión en cuanto a la relación de la producción agropecuaria sostenible y la conservación de humedales, la cual se identificó en la ejecución de un Plan General de Manejo Refugio Nacional de Vida Silvestre Mata Redonda, ejecutado por el Centro Mesoamericano de Desarrollo 
Revista Universidad en DiÁlogo • Vol. 7, N. ², Julio-Diciembre, 2017, pp. 11-23

ISSN 2215-2849 • EISSN: 2215-4752

DOI: http://dx.doi.org/10.15359/udre.7-2.1

Sostenible del Trópico Seco (CEMEDE) de la UNA, en el 2013. En el presente documento se analiza el interés mostrado por la comunidad a lo largo del proyecto y su experiencia al implementar el uso de tecnologías alternativas agropecuarias sostenibles como medidas de adaptación al cambio climático en sus fincas, las dificultades y logros que enfrentaron como productores. El proyecto consistió en un trabajo participativo entre la academia, la comunidad y la administración del Área de Conservación Tempisque (ACT), donde se promovió la utilización de tecnologías sostenibles para la conservación de los ecosistemas de humedal.

Palabras clave: humedal, fincas pecuarias integradas, áreas protegidas, ganadería sostenible, silvicultura.

Abstract. The main objective of this investigation was to study the receptivity, application and usefulness of small farmers in the framework of the project Establishment and Management of Integrated Farms. The project started in 2014 and finished in December 2016; it was conducted in the ecosystem of Mata Redonda wetland, Rosario de Nicoya, Costa Rica. The project was born thanks to the results of the relation between the sustainable agricultural production and the conservation of wetlands, which were identified in the execution of the Plan General de Manejo Refugio Nacional de Vida Silvestre Mata Redonda (General Plan for Management of Mata Redonda National Wildlife Refuge) by the Centro Mesoamericano de Desarrollo Sostenible del Trópico Seco (Mesoamerican Center for Sustainable Development of the Dry Tropics- CEMEDE) in 2013. This paper analyzes the interest expressed by the community during the implementation of the project. It also analyzes the farmers' experience when implementing alternative, sustainable, agricultural technologies as measures for adaptation to the climate change in their farms, as well as the difficulties and achievements these producers faced. The project consisted of a participatory work between the academy, the community and the administration of the Área de Conservación Tempisque (ACT-Tempisque Conservation Area), which promoted the implementation of sustainable technologies for the conservation of the wetland ecosystems.

Keywords: wetland, integrated livestock farms, protected areas, sustainable livestock, forestry.

\section{Introducción}

El Ministerio de Agricultura y Ganadería (MAG) incorporó en la Política de Estado para el Sector Agroalimentario y el Desarrollo Rural Costarricense 2010-2021 un pilar de trabajo denominado Gestión de Territorios Rurales y Agricultura Familiar, del que se deriva el Plan Sectorial de Agricultura Familiar 
2011-2014. En este se definió un eje transversal de cambio climático dirigido al fomento de los sistemas alternativos de producción, tales como agricultura orgánica, manejo integrado de cultivos, los sistemas silvopastoriles y la producción sostenible (IICA, 2017). En el presente artículo se va a hablar de la experiencia en la receptividad obtenida de los pequeños ganaderos en un proyecto de investigación de establecimiento de fincas integrales como una medida de adaptación al cambio climático de ganadería en ecosistema de humedal.

La investigación se ejecutó en Rosario de Nicoya, donde se encuentra el Refugio de Área Silvestre Protegida de Mata Redonda, que mediante el Decreto Ejecutivo N²2764-MIRENEM, en 1994, se declaró Refugio Nacional de Vida Silvestre, propiedad del Estado. En este decreto, se establece que este Refugio puede ser utilizado para pastoreo y abrevadero de especies bovinas, equinas y porcinas. Por lo anterior, los ganaderos de la zona en el día llevan su ganado a pastar a la laguna. Además, el 1 de setiembre de 2002, Mata Redonda se designó como un anexo al sitio Ramsar del Parque Nacional Palo Verde por ser parte de un sistema de hábitat para las aves acuáticas de la cuenca baja del Tempisque. Se considera el sitio de mayor importancia en el ámbito nacional para la protección del jabirú (Jabirú mycteria), ave acuática en inminente peligro de extinción (ACT, 2016). En el caso de Mata Redonda sus vecinos inmediatos se dedican principalmente a la actividad ganadera, por lo tanto, es necesario desarrollar, en forma conjunta con los grupos productores, alternativas de manejo pertinentes para una ganadería sostenible en zonas aledañas a humedales.

\section{Marco teórico}

Según la Convención Ramsar (1971), un humedal es una zona de la superficie terrestre que está temporal o permanentemente inundada, regulada por factores climáticos y en constante interrelación con los seres vivos que lo habitan (UNESCO, 1971). En la cuenca baja del río Tempisque los ecosistemas de humedal son característicos del lugar, por lo que se consideran sitios Ramsar de interés mundial declarados por dicha Convención. Además, son Patrimonio Nacional del Estado en la categoría de Área Silvestre Protegida (ASP) y administrada por el Sistema Nacional de Áreas de Conservación (SINAC) del Ministerio de Ambiente y Energía (MINAE). Este reconocimiento mundial claramente requiere la ejecución de acciones apropiadas por parte de las personas que se encuentran en zonas aledañas a estas ASP, en especial quienes ejecutan actividades productivas que, de una $u$ otra manera, impactan el ecosistema del humedal. 
ReVista Universidad en DiÁlogo • Vol. 7, N. ํㄹ 2, Julio-Diciembre, 2017, pp. 11-23

ISSN 2215-2849 • EISSN: 2215-4752

DOI: http://dx.doi.org/10.15359/udre.7-2.1

Siendo los humedales un filtro de depuración de aguas superficiales y un captador de dióxido de carbono, su protección debe ser participativa, donde se involucre tanto la comunidad como las entidades estatales (Artavia, 2004).

La contaminación de las aguas superficiales y subterráneas por parte de las excretas bovinas y la lixiviación de agroquímicos, además de la erosión y compactación de los suelos, son parte de los principales impactos negativos que genera la actividad ganadera a los humedales. Esta problemática, sumada a la emisión de metano, gas efecto invernadero proveniente de la digestión de los rumiantes, justifica la importancia de generar alternativas de manejo sostenible para la ganadería en zonas aledañas a los humedales.

En el caso de Mata Redonda, sus pueblos vecinos inmediatos se dedican, principalmente, a la actividad ganadera, por lo tanto, es necesario desarrollar, en forma conjunta con los grupos productores, alternativas de manejo pertinentes para una ganadería sostenible en zonas aledañas a humedales como medida de adaptación al cambio climático.

El cambio climático es una realidad planetaria que se debe afrontar con acciones concretas que permitan la adaptación del ser humano ante dichos cambios; al mismo tiempo que se reduce la vulnerabilidad de la sociedad ante eventos climáticos extremos. Según UNED-SINAC (2012), la región Chorotega y específicamente la cuenca baja del río Tempisque son uno de los sitios afectados por el calentamiento planetario, por lo cual presentan indicios de desertificación que se pueden agravar a futuro, si no se ejecutan acciones de mitigación.

El manejo integrado de fincas pecuarias es una forma de producción sostenible que contempla el uso de tecnologías alternativas limpias, sencillas y de bajo costo; siempre trata de cerrar e internalizar ciclos y flujos de nutrientes y permite mantener un sistema de producción dinámico, donde los excedentes vuelven al sistema en forma de ingresos económicos para el sujeto productor y su familia. Estas tecnologías alternativas ecoeficientes de producción son las que se implementaron con el proyecto en Rosario de Nicoya.

El modelo de fincas integrales trabaja con base en el aprovechamiento de los recursos naturales y el balance de las funciones del agroecosistema, 
introduciendo prácticas ambientalmente coherentes y más saludables para las familias productoras, así como para la comunidad. La finca integral combina la conservación del suelo y de la biodiversidad, el ciclaje de nutrientes y la regulación de plagas, la producción de alimentos y el tratamiento de desechos, en esferas que interactúan y hasta cierto punto se superponen, reconociendo que la producción agropecuaria integral está incorporada al paisaje y, como este, tiene múltiples funciones diversas y complejas (Navarro, 2014).

Dentro de las principales alternativas con las que se cuenta para el manejo de fincas integradas pecuarias, podemos mencionar la cosecha y almacenamiento de agua de lluvia en reservorios, biodigestores para el manejo de excretas y aguas residuales, bancos forrajeros y ensilajes para la suplementación animal en épocas críticas, silvopasturas para la reducción del estrés calórico de los animales y abonos orgánicos para la fertilización de pasturas. Dichas tecnologías alternativas ecoeficientes contribuyen, en gran medida, a reducir la presión de la ganadería sobre los humedales, al contar los sectores ganaderos con las fuentes de alimentación e hidratación para sus animales dentro de sus propias fincas (Botero y Preston, 1987).

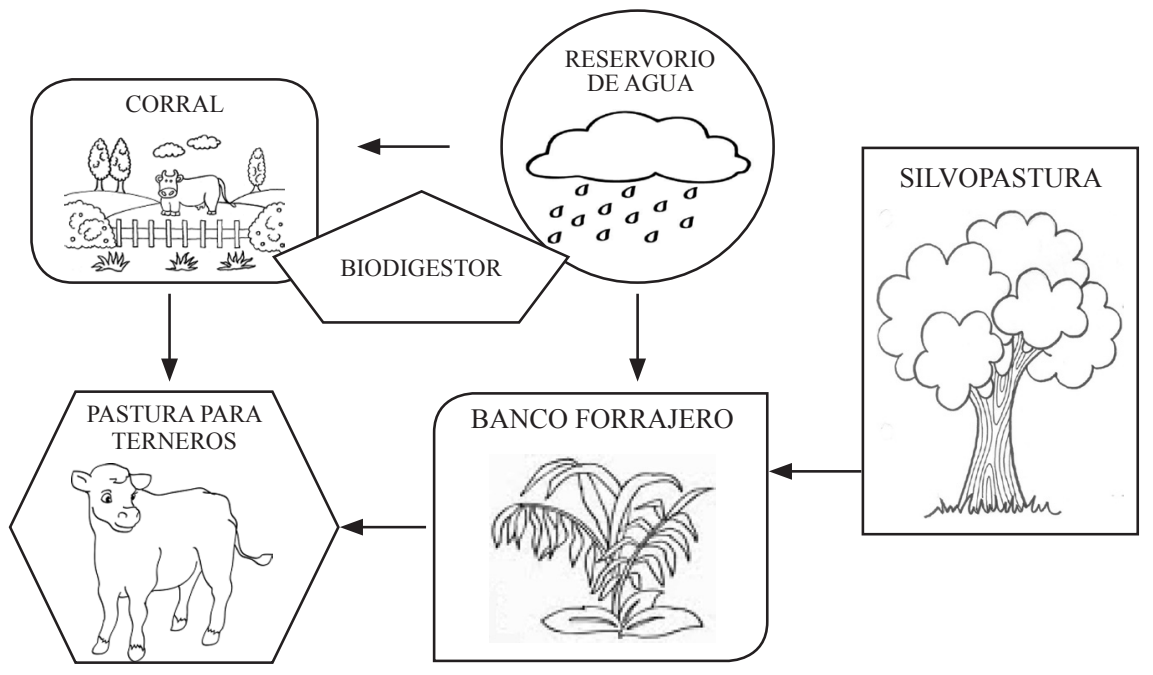

Figura 1. Modelo de fincas integrales. Elaboración propia (2017). 
Revista Universidad en Diálogo • Vol. 7, N. ${ }^{\circ} 2$, Julio-Diciembre, 2017, pp. 11-23

ISSN 2215-2849 • EISSN: 2215-4752

DOI: http://dx.doi.org/10.15359/udre.7-2.1

Los biodigestores son estructuras cerradas herméticamente por donde pasan los desechos orgánicos para la generación de biogás a través de la fermentación. Este sistema en instalaciones ganaderas permite la descontaminación de un $80 \%$ de las excretas y aguas residuales de los animales (Botero y Preston, 1987). Además, el efluente líquido excedente del proceso de fermentación en el biodigestor puede ser utilizado para la fertilización de pasturas y forrajes de corte.

Los bancos forrajeros son cultivos de plantas con alto contenido de biomasa, los cuales permiten, por medio de la corta o cosecha periódica, la suplementación del ganado en épocas críticas, como la época seca en la región Chorotega (Orozco, 2009). Cabe recalcar la relación que debe tener este componente de la finca con las tecnologías de reservorios y biodigestores, ya que es de ahí de donde saldrá el agua y el fertilizante, respectivamente, para una alta producción de biomasa en el banco forrajero.

El forraje debe ser conservado para poder utilizarse cuando más se necesite, por lo que la técnica que mayor se adapta a esta necesidad es la preparación de ensilajes, la cual consiste en la corta y picadura del forraje para su posterior compactación y tapado, con lo que se evita la precia y el contacto con el oxígeno. Esto asegura una fermentación correcta del forraje y, por lo tanto, su conservación prolongada a disposición del momento en que se requiera (Méndez, 2008).

\section{Metodología}

\section{Área de estudio}

La comunidad de Rosario se encuentra en la provincia de Guanacaste, en el cantón de Nicoya y pertenece al distrito $3^{\circ}$ San Antonio, se ubica a unos 36 $\mathrm{km}$ de Nicoya (observe la figura 1); cuenta con una población de 628 habitantes; tiene servicios públicos como electricidad, telefonía, agua potable y transporte. Está conformada, en su mayoría, por grupos pequeños y medianos de productores de ganado, dedicados principalmente a la ganadería bovina, de cerdos y gallinas para autoconsumo. El 30\% de la población posee terrenos entre 2 y 10 has. Se cultiva para autoconsumo maíz, frijoles y cuadrada. Una de las principales fuentes de recursos es la Laguna de Mata Redonda, en la que principalmente se dan la pesca de tilapia; la cacería de piches; la recolección de chan, paste uvita, carao, semillas de guácimo y de tempisque. Se corta madera principalmente para postes de cerca. 


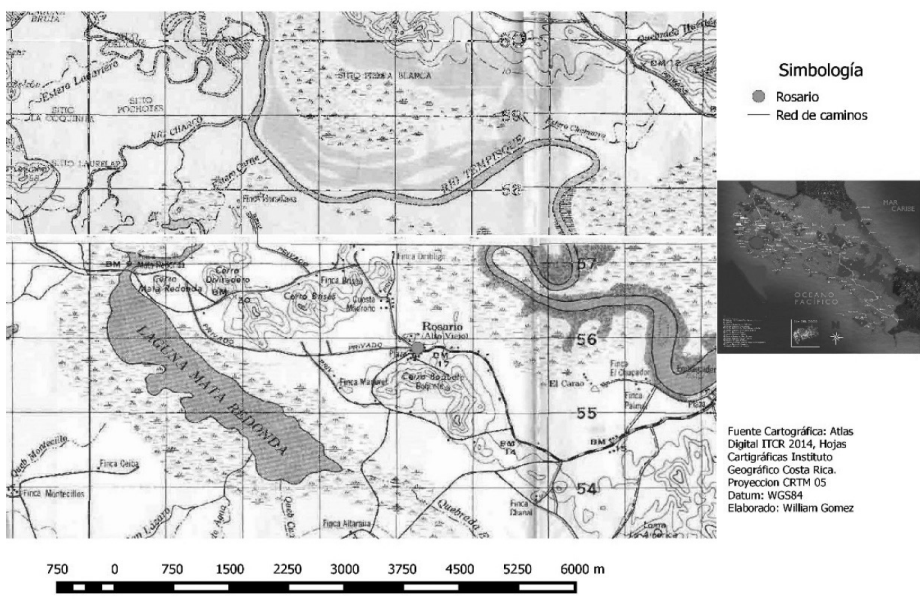

Figura 2. Ubicación geográfica de Rosario de Nicoya (Gómez, 2017).

Para analizar la receptividad, aplicación y utilidad del establecimiento y manejo de fincas pecuarias integradas por parte de los grupos productores ganaderos de zonas aledañas a los ecosistemas de humedal se utilizó la sistematización de experiencias propuesta en Práctica y teoría para otros mundos posibles, de Hugo Jara. En esta obra se define la sistematización de experiencias a partir del análisis de otras definiciones y de la búsqueda de elementos fundamentales:

La sistematización es aquella interpretación crítica de una o varias experiencias que, a partir de su ordenamiento y reconstrucción, descubre o explicita la lógica y el sentido del proceso vivido en ellas: los diversos factores que intervinieron, cómo se relacionaron entre sí y por qué lo hicieron de ese modo. La sistematización de experiencias produce conocimientos y aprendizajes significativos que posibilitan apropiarse críticamente de las experiencias vividas (sus saberes y sentires), comprenderlas teóricamente y orientarlas hacia el futuro con una perspectiva transformadora $(2012$, p. 71$)$.

Además, el autor pone el énfasis en que la sistematización ha de servir para comprender las experiencias y poder mejorarlas, para compartir aprendizajes con otras experiencias, para fomentar la reflexión teórica con conocimientos de la propia práctica, para poder orientar los grandes programas institucionales desde los conocimientos generados en las experiencias particulares y para fortalecer la identidad colectiva (Jara, 2012). 
Revista Universidad en Diálogo • Vol. 7, N. ํㅡㄹ Julio-Diciembre, 2017, pp. 11-23

ISSN 2215-2849 • EISSN: 2215-4752

DOI: http://dx.doi.org/10.15359/udre.7-2.1

En el proyecto se programaron ocho talleres en los siguientes temas:

- Bloques multinutricionales

- Nutrición de rumiantes

- Inocuidad del ordeño

- Procesamiento de lácteos

- Elaboración de ensilajes

- Abonos orgánicos

- Condición corporal de bovinos

- Manejo de animales doble propósito

Gracias a la excelente participación de los grupos productores en estos talleres, al interés mostrado y a su solicitud, se impartieron siete talleres más, los cuales no estaban contemplados en la formulación del proyecto:

- Establecimiento de biodigestores

- Condiciones para la aplicación de buenas prácticas agropecuarias dentro de las ASP

- Registros productivos para el manejo del hato

- Manejo nutricional del hato

- Planes de fertilización forrajera sostenible

- Programa de control sanitario del hato

- Manejo de aguas residuales en instalaciones lecheras o semiestabulados de carne

Para la ejecución de los talleres se realizaron dos sesiones de trabajo por tema, ya que una sesión era teórica y otra práctico-participativa, además en cada una de las sesiones se les asignaban tareas. Luego de cada taller tenían un plazo de un mes para ejecutar las tareas; un mes después de cada taller se realizó una gira de campo, en la cual se visitaba la finca de cada persona participante para evaluar la ejecución de la tarea asignada, aclarar cualquier duda y verificar si lo programado se ejecutó. Es importante recalcar que, en el mes en que se ponía en práctica la asignación, el equipo técnico del proyecto le brindó apoyo y asesoría a cada productor o productora, con visitas semanales para crear espacios de análisis que permitieran retroalimentar el proceso de trabajo e ir identificando la receptividad, utilidad y 
uso. Lo descrito anteriormente permitió monitorear e identificar el interés de los sujetos productores participantes y la importancia que le daban a la implementación de la tecnología en su finca y cuántos días después de los talleres se aplicaba el conocimiento adquirido. Al mismo tiempo, se identificaban las condiciones/limitaciones al aplicar el conocimiento adquirido.

\section{Discusión de los resultados}

El análisis de los resultados obtenidos en el proyecto permitió determinar que los sujetos productores de la Asociación de Pequeños Ganaderos de la Laguna de Mata Redonda están utilizando en sus fincas tecnologías sostenibles para la conservación de los ecosistemas de humedal, y se han adaptado exitosamente a los efectos del cambio climático. Este proyecto puede servir de ejemplo y como punto de partida para otras poblaciones aledañas a ecosistemas de humedal que tengan entre sus principales actividades económicas la ganadería.

El proyecto tuvo una relevancia sorpresiva e inesperada en cuanto a la receptividad de los ganaderos, ya que cuando se enfrentó la sequía del 2015 fue cuando el grupo se fortaleció más, se incorporaron más personas productoras y por solicitud del grupo se les apoyó con la creación de la Asociación de Pequeños Ganaderos de la Laguna de Mata Redonda. Al inicio del proyecto estaban involucradas ocho familias, al finalizar el proyecto había quince familias.

Complementario al proyecto, se lograron grandes avances en la comunidad gracias a la excelente receptividad de los grupos ganaderos de Rosario. Estos avances, que no estaban establecidos dentro de los objetivos, son:

- La integración de jóvenes al proyecto: la mayoría de estos jóvenes son hijos, hijas o familiares de los sujetos productores con los que se trabaja en el proyecto y se fueron acercando voluntariamente a los talleres de capacitación, lo que le da un realce y ratifica la continuidad de la producción sostenible en las fincas, tomando en cuenta el cambio generacional. Además de que les brindó a estos grupos de jóvenes la oportunidad de tener una entrada económica, que antes no tenían, lo cual favorece que no deban migrar a Nicoya o a la capital en busca de mejores oportunidades económicas para ayudar a sus familias. 
ReVista Universidad en DiÁlogo • Vol. 7, N. ํㄹ 2, Julio-Diciembre, 2017, pp. 11-23

ISSN 2215-2849 • EISSN: 2215-4752

DOI: http://dx.doi.org/10.15359/udre.7-2.1

- Donaciones que recibió el proyecto por diferentes personas de la comunidad para el proceso de siembra (maquinaria, mano de obra para la preparación del terreno y semilla) reflejan el interés de la comunidad en el proyecto.

- Conformación de la Asociación de Productores Ganaderos de la Laguna de Mata Redonda, ya que gracias al proyecto formaron una agrupación organizada.

- Gracias al trabajo del grupo, una persona de la comunidad se ofreció a donar un terreno para la construcción del Centro de Acopio de Leche para la Asociación.

- Como ya era un grupo bien consolidado y conocido en la zona, cuando la Comisión de Emergencias y el MAG brindaron la ayuda de donación de insumos agropecuarios para enfrentar la sequía en el 2015, la Asociación fue uno de los primeros grupos a los cuales se les donaron las pacas de pasto y otros productos.

- Al ser un grupo bien conformado, han logrado ayudas y fondos de otras instituciones que llegan a la zona y empiezan a trabajar con el grupo, como el Ministerio de Trabajo, el Ministerio de Agricultura y Ganadería, el Ministerio de Ambiente y Energía y la Cámara de Ganaderos de Nicoya.

- El grupo mejoró la relación y se integraron a trabajar en equipo con el Área de Conservación Tempisque (ACT) y a apoyar en la rehabilitación de la Laguna de Mata Redonda.

- Con el trabajo realizado durante los años del proyecto, se identificó la falencia de capacidades para la creación de un centro de acopio de leche, por lo que se redactó entre la academia y la Asociación de Productores un proyecto para trabajar en estas mismas en el 2017-2018. Este proyecto concursó por financiamiento y obtuvo presupuesto para su realización.

\section{Conclusiones y recomendaciones}

Una de las principales acciones por las que se trabajó con la comunidad de Rosario de Nicoya fue por la preocupante situación ante el cambio climático, el cual ha originado sequías que han tenido alto impacto en los sistemas productivos de la comunidad. Por lo que el modelo de fincas integrales en el que se orienta a los productores a cambiar de los modelos 
tradicionales de producción a modelos sostenibles lleva a la implementación de tecnologías sostenibles para la conservación. Las cuales se enfocan en la mejora de las condiciones en las que se utilizan los recursos, generando un beneficio no solo para el productor y su finca, sino que también contempla acciones de mitigación y adaptación a los efectos que el cambio climático ha ido generando y tomando previsiones hacia afectaciones futuras.

Las acciones implementadas en esta región son un modelo de agricultura sostenible que puede ser replicado en diferentes regiones y principalmente en los sectores aledaños a la Laguna de Mata Redonda, en la cual se benefician productores de otros poblados como Corral de Piedra y Pozo de Agua. Pero este modelo de fincas integrales, al ser estructural con el sistema de producción, se puede expandir a otras zonas aparte de la región Chorotega o de zonas de bosque seco o bajura, dejando abierta la posibilidad de adaptación en diferentes sectores productivos y ecosistemas.

Este caso presenta la particularidad de la utilización de una zona protegida como la Laguna de Mata Redonda para el pastoreo por parte de los vecinos; este es un caso peculiar, ya que en muy pocas condiciones se permite esta asociación de productores con los recursos naturales protegidos. Pero es una relación simbiótica de apoyo entre ambas partes, ya que los ganaderos pueden aprovechar las pasturas y el agua de la laguna; pero por otro lado estas acciones permiten mantener limpio el espejo de agua de la laguna para el paso de aves migratorias.

Las relaciones entre las comunidades y los funcionarios del Área de Conservación Tempisque no estuvieron en buenos términos durante un tiempo. Pero la implementación de estas prácticas ha permitido que esta situación cambie y que en la actualidad ambos bandos trabajen en conjunto para la preservación y conservación de los recursos naturales en la zona. Esta asociación no solo promueve que se siga con el modelo de producción de leche y conservación de la finca, ya que es un caso en el que se demuestra que el trabajo cooperativo entre la Administración pública y productores privados puede llevar a esfuerzos en beneficio de ambas partes y principalmente de la conservación de los recursos naturales.

Un producto esencial que se logró es la integración de productores que conforman un grupo organizado y que siguen una meta común, en este caso la implementación de los modelos de fincas integrales y la preservación de los recursos naturales. 
Revista Universidad en Diálogo • Vol. 7, N. ํㅡㄹ Julio-Diciembre, 2017, pp. 11-23

ISSN 2215-2849 • EISSN: 2215-4752

DOI: http://dx.doi.org/10.15359/udre.7-2.1

El hecho de que estos productores se estén organizando para establecer un Centro de Acopio de Leche, con el cual mejorarán sus condiciones en los ingresos por la venta de productos lácteos, es un logro externo que indica que con los incentivos adecuados y la unión de diferentes entidades se pueden hacer grandes cosas en beneficio de los sectores involucrados. En este caso es un beneficio a nivel familiar integral, ya que se unen diferentes generaciones en busca del desarrollo y mejora de sus condiciones de vida.

\section{Referencias}

ACT. (2016). Área de Conservación Tempisque (ACT). Recuperado de www.actempisque.org/rnvsmataredonda

Artavia, M. (2004). Guía para la formulación de planes de manejo de Áreas Silvestres Protegidas. Ministerio de Ambiente y Energía. San José, Costa Rica.

Botero, R. y Preston, T. (1987). Biodigestor de bajo costo para la producción de combustible y fertilizante a partir de excretas. EARTH. San José, Costa Rica.

Gómez, W. (2017). Ubicación geográfica Rosario de Nicoya. Atlas digital de Costa Rica. Proyección CRTM05. Datum WGS84. CEMEDE, UNA.

IICA (2017). La agricultura tropical frente al cambio climático, Costa Rica Carbono Neutral 2021. Recuperado de http://www.infoagro. net/programas/regatta/pages/actividades/Compendio_Es.pdf

Jara, H. (2012). Práctica y teoría para otros mundos posibles. San José, Costa Rica: Centro de Estudios y Publicaciones Alforja.

Méndez, J. (2008). Agrocadena de la ganadería bovina de carne de la región Chorotega. Manual de recomendaciones para el manejo sostenible de la ganadería bovina de carne en la región Chorotega. Ministerio de Agricultura y Ganadería. San José, Costa Rica.

Navarro, A. (2014). Fincas integrales: aportes a los servicios ecosistémicos y a la calidad de vida de las familias. Revista de Agroecología, 30(3), 29-31.

Orozco, E. (2009). Estrategias de manejo y suplementación para ganado durante la época seca. San José, Costa Rica: MAG/INTA. 
UNED-SINAC. (2012). Plan General de Manejo del Refugio Nacional de Vida Silvestre Mata Redonda. Herramienta de Manejo Adaptativo y Planificación Estratégica. Universidad Estatal a Distancia. Sistema Nacional de Áreas de Conservación, Área de Conservación Tempisque. Nicoya, Costa Rica. 170p.

UNESCO. (1971). Convención Ramsar. Convención Relativa a los Humedales de Importancia Internacional. 